\title{
Onkologische Therapie in der Gastroenterologie. Was ist evidenzbasiert? Perspektiven?
}

\section{Oncological Therapy in Gastroenterology. What Is Evidence-Based? Perspectives?}

\author{
Gesprächsleiter: G. Adler, Ulm \\ Teilnehmer: W. O. Bechstein, Frankfurt/M. M. Heike, Dortmund P. Hohenberger, Berlin \\ H. Neuhaus, Düsseldorf R. Sauer, Erlangen
}

Frage 1: Welche Polypen in Ösophagus und Magen sollten mukosektomiert werden? Welche Technik ist die beste? Gibt es unterschiedliche Strategien abhängig vom Alter der Patienten?

Neuhaus: Im Ösophagus erscheint die Mukosektomie angezeigt bei fokalen Läsionen mit hochgradiger intraepithelialer Neoplasie oder einem auf die Mukosa beschränkten Frühkarzinom im Plattenepithelbereich oder in einem Barrett-Segment. Im Magen ergibt sich eine Indikation bei Adenomen oder mukosalen Karzinomen. Nach endoskopischen und endosonographischen Kriterien sollte dabei kein Hinweis auf eine über die Mukosa hinausgehende vertikale Tumorinvasion vorliegen. Eine lokale Therapie erscheint kurativ nur ausreichend bei histologischer Bestätigung einer fehlenden Infiltration der Submukosa sowie sichergestellter kompletter Resektion. In allen anderen Fällen, d.h. bei nicht sichergestellter vollständiger Entfernung lateraler Anteile, multifokalen Läsionen, besonders bei einem Long-Segment-Barrett, sowie oberflächlicher Invasion der Submukosa $(<0,5 \mathrm{~mm})$ muss interdisziplinär individuell entschieden werden, ob die Mukosaresektion eine ausreichende Therapie darstellt. Dabei muss das Risiko von Lokalrezidiven oder von Lymphknotenmetastasen bei Patienten im höheren Alter oder bei Begleiterkrankungen im Einzelfall dem Risiko einer Ösophagektomie bzw. Gastrektomie gegenübergestellt werden. Die endoskopische Mukosaresektion sollte in einem Zentrum erfolgen. Bei entsprechender Expertise sind die verschiedenen Verfahren sicher und in ihrer Effektivität vergleichbar, wobei allerdings nur vereinzelt kontrollierte Studien vorliegen. Neu entwickelte Methoden ermöglichen besonders im Magen auch eine En-bloc-Resektion größerer Areale. Sie bedürfen allerdings, insbesondere in westlichen Ländern, noch einer umfangreichen Evaluation.
Bechstein: Im Gegensatz zum Kolon treten Polypen im Ösophagus und Magen selten auf. Ein häufigeres klinisches Problem stellen lokale Schleimveränderungen im Ösophagus bzw. gastroösophagealen Übergang im Rahmen einer BarrettMetaplasie dar. Verschiedene Techniken der endoskopisch mukosalen Resektion sind gebräuchlich, angefangen von Abtragung mit der Elektroschlinge über die Platzierung eines Gummirings (wie zur Gummibandligatur von Varizen gebräuchlich) zur Schaffung eines «Pseudopolypen» und anschließenden Abtragung bis zur «Suck-and-Cut»-Methode, bei der eine durchsichtige Saugglocke den veränderten Schleimhautbezirk ansaugt, so dass dieser gleich anschließend abgetragen werden kann. Da die Techniken untersucher- und erfahrungsabhängig sind, gibt es keine gesicherten Erkenntnisse darüber, welche Technik möglicherweise die beste wäre [1].

Heike: Entsprechend der neuen Wien-Klassifikation werden mukosale polypöse Raumforderungen der Ösophaguswand nach $\mathrm{NaCl}$-Unterspritzung mukosektomiert. Voraussetzung ist der Ausschluss eines invasiven, in die Submukosa infiltrierend wachsenden Karzinoms, das in der Regel operativ entfernt wird. Nach der neuen Wien-Klassifikation entspricht das der Kategorie 4 mit den Untergruppen 4.1 bis 4.4. In Einzelfällen wird bei alten Patienten in Abhängigkeit vom Operationsrisiko auch ein submukosal infiltrierendes Karzinom endoskopisch nach $\mathrm{NaCl}$-Unterspritzung abgetragen und gegebenenfalls nachbehandelt. In der Regel werden diese Patienten jedoch auch im höheren Lebensalter operiert.

Hohenberger: Prinzipiell sollten alle Polypen in Ösophagus und Magen im Sinne einer Exzisionsbiopsie entfernt werden, wenn sich bioptisch Dysplasien nachweisen lassen. Rein hyperplastische Polypen sind ohne Krankheitswert und kön-

\begin{tabular}{ll}
\hline KARGER & ( ) 2004 S. Karger GmbH, Freiburg \\
$\begin{array}{l}\text { Fax +49 761 4520714 } \\
\begin{array}{l}\text { E-mail Information@Karger.de } \\
\text { www.karger.com }\end{array}\end{array}$ & $\begin{array}{l}\text { Accessible online at: } \\
\text { www.karger.com/cga }\end{array}$
\end{tabular}


nen belassen werden. Es gibt keine Festlegung bzw. Daten welche endoskopische Resektionstechnik die beste ist, so dass diejenige angewendet werden sollte, mit der der Endoskopiker am besten vertraut ist, um eine komplette Resektion zu erzielen. Für größere Tumoren der Magenwand, (nicht Submukosa, z.B. GIST) kann ein kombiniertes endoskopisch-laparoskopisches Verfahren vorteilhaft sein.

\section{Frage 2: Welchen Informationsgewinn erzielt man durch den Einsatz von Färbeverfahren bei der Endoskopie im Ösophagus?}

Bechstein: Die Barrett-Metaplasie des Ösophagus ist als Präkanzerose zu betrachten, das Risiko für die Entwicklung eines Adenokarzinoms des Ösophagus im Vergleich zur Normalbevölkerung ist 30- bis 40-fach erhöht. Die Entdeckung der Barrett-Metaplasie zur gezielten Biopsie und gegebenenfalls Mukosektomie hat daher einen hohen klinischen Stellenwert. Meist wird Methylenblau verwendet - ideal in Zusammenhang mit einem vergrößerten Sichtfeld. Die MethylenblauChromoendoskopie vermag mit einer Sensitivität von $84,8 \%$ und einer Spezifität von 91,7\% eine intestinale Metaplasie im Ösophagus zu entdecken [2]. In einer Kohortenstudie mit 975 Patienten konnte bei 35 Patienten in 114 von 130 Methylenblau-gefärbten Schleimhautbezirken bei gezielter Biopsie eine Barrett-Metaplasie nachgewiesen werden [3]. Es gibt jedoch keine Evidenz, dass der routinemäßige Einsatz von Chromoendoskopie die Prognose des Patienten mit Barrett-Metaplasie verändert.

Heike: Von den möglichen Färbeverfahren im Ösophagus hat sich bei uns die Färbung mit Lugol'scher Lösung und in Einzelfällen deren Kombination mit Methylenblau beim BarrettÖsophagus vor geplanter Mukosektomie durchgesetzt. Die Färbung mit 1,5\% Essigsäure scheint zur Diagnostik und Überwachung des Barrett-Ösophagus ebenfalls eine sehr geeignete Methode zu sein.

Hohenberger: Färbeverfahren wie Methylenblau nach ACCSpülung dienen der besseren Kontrastierung unruhiger Schleimhautabschnitte und haben ihren Stellenwert bei der Barrett-Schleimhaut oder der Frühkarzinomsuche. Lugol'sche Lösung hat sich für die Demarkation dysplastischer Areale bewährt.

Neuhaus: Bei Verdacht auf eine frühe Neoplasie im Plattenepithelbereich ermöglicht die Anfärbung mit Lugol'scher Lösung eine bessere Abgrenzung der lateralen Ausdehnung einer Läsion sowie die Detektion multifokaler Areale. Bei einem Barrett-Segment verspricht die Chromoendoskopie mit Methylenblau eine zuverlässigere Erkennung von neoplastischen Bezirken. Die Ergebnisse kontrollierter Studien sind jedoch kontrovers und ergaben nur teilweise eine Überlegenheit gegenüber einer Standardendoskopie. Die Chromoendoskopie ist somit empfehlenswert, bedarf jedoch weiterer Evaluation hinsichtlich der Färbetechniken und der Interpretation der Befunde.

\section{Frage 3: Was ist der Stellenwert der neoadjuvanten Radiochemotherapie beim Ösophaguskarzinom? Welche Auswirkungen hat diese Therapie auf das Operationsergebnis?}

Sauer: Die neoadjuvante Radiochemotherapie des Ösophaguskarzinoms führt in $60 \%$ der Fälle zu objektiven Remissionen, in etwa $35 \%$ gar zu histologisch konfirmierten kompletten Remissionen. Dies erleichtert die Operationsverfahren und erlaubt sogar bei T3- und T4-Karzinomen in etwa $80 \%$ $\mathrm{R} 0$-Resektionen. Inwieweit sich dies allerdings in ein verbessertes, Lokalrezidiv-freies Überleben übersetzt oder gar in eine Senkung der Rate an Fernmetastasen, ist bisher nicht abschließend geklärt.

Bechstein: $\mathrm{Zu}$ unterscheiden ist zunächst zwischen dem Plattenepithelkarzinom des Ösophagus und dem Adenokarzinom des Ösophagus. Gleichwohl konnte bei beiden Entitäten durch mehrere randomisierte Studien gezeigt werden, dass eine präoperative Radiochemotherapie ein effektives «downstaging» ermöglicht, bis hin zu einem im Operationspräparat nicht mehr nachweisbaren Tumor (Typ T0); gleichzeitig wird die chirurgische Radikalität durch einen höheren Anteil von R0-resezierten Patienten erhöht, der Anteil R1-resezierter Patienten wird signifikant geringer. Ob die Langzeitergebnisse hierdurch verbessert werden, ist weiter Gegenstand kontroverser Diskussion [4-6]. Ebenso wird kontrovers diskutiert, ob die präoperative Radiochemotherapie zu einer erhöhten perioperativen Mortalität und Morbidität führt im Vergleich zu Patienten, die direkt operiert werden.

Heike: Bisher konnte in Studien nicht eindeutig nachgewiesen werden, dass die neoadjuvante Radiochemotherapie gefolgt von der Tumorresektion Überlebensvorteile gegenüber der primären Resektion oder gegenüber einer definitiven Radiochemotherapie ohne nachfolgende Resektion erbringt. In drei randomisierten Studien [7-9] deuten sich jedoch Überlebensvorteile gegenüber einer primären Resektion an. Ein häufiges Problem der neoadjuvanten Radiochemotherapie ist eine erhöhte perioperative Mortalität, die abhängig vom Bestrahlungsprotokoll, der Strahlendosis und der Patientenauswahl zu sein scheint. Bei $25-30 \%$ der Patienten werden durch die neoadjuvante Radiochemotherapie pathologisch komplette Remissionen und dadurch eine deutliche Prognoseverbesserung erzielt.

Hohenberger: Eine neoadjuvante Radiochemotherapie ist bei Ösophaguskarzinomen des proximalen und mittleren Drittels ein effektives Verfahren, lokal fortgeschrittene Tumoren (T3 
$\mathrm{NX}, \mathrm{TX} \mathrm{N}+$ ) zu kontrollieren. Allerdings ist die perioperative Morbidität und Mortalität nach Radiochemotherapie deutlich höher. Nicht definitiv geklärt ist die Frage, ob Patienten mit einem Ansprechen auf eine neoadjuvante Radiochemotherapie durch Resektion des tumortragenden Ösophagusabschnitts und seiner Lymphabflusswege oder durch zusätzliche Boost-Bestrahlung besser kontrolliert werden können. Tumoren in T2-N0-Stadien sollten primär operiert werden. Dies gilt auch für T1-Tumoren, die nicht sicher durch endoskopische Maßnahmen beherrscht werden können.

Neuhaus: Eine neoadjuvante Chemotherapie oder Radiochemotherapie sollte sowohl bei einem Plattenepithel- als auch bei einem Barrett-Karzinom des Ösophagus zumindest im Tumorstadium IIB und III erwogen werden. Die Interpretation der unterschiedlich konzipierten kontrollierten Studien ist jedoch schwierig, und die Ergebnisse sind nicht eindeutig. Wahrscheinlich gelingt eine Verbesserung der lokalen Tumorkontrolle, möglicherweise auch eine Verlängerung der Überlebenszeit. Die Wahrscheinlichkeit einer distalen Metastasierung scheint dagegen nicht beeinflusst zu werden. In Abhängigkeit vom Bestrahlungsprotokoll kann sich die postoperative Mortalität erhöhen. Die Entscheidung zu einer neoadjuvanten Therapie sollte interdisziplinär getroffen werden; wünschenswert ist ein Einschluss der Patienten in laufende kontrollierte Studien.

\section{Frage 4: Wie viel operative Radikalität ist beim Magenkarzinom notwendig? Ist die adjuvante Radiochemotherapie der neue Standard nach operativer Therapie eines Magenkarzinoms?}

Sauer: Die operative Radikalität richtet sich nach dem Karzinomtyp und dem Tumorstadium. Die Lymphadenektomie des Kompartement 1 (D1-Resektion) gilt als Standard; die D2-Resektion erweist sich bei einer Subgruppe von Patienten (intestinaler Typ, T3-Karzinome) als vorteilhaft. Die adjuvante und neoadjuvante Radiochemotherapie wird vielerorts bei Karzinomen des gastroösophagealen Übergangs empfohlen. Die ermutigenden Resultate der postoperativen Radiochemotherapie beim Magenkarzinom der amerikanischen Southwest Oncology Group [10] werden in Europa aber wegen der Verletzung operativ-onkologischer Radikalitätsprinzipien nicht akzeptiert.

Bechstein: Zwei randomisierte Studien zum Vergleich einer D1Lymphadenektomie mit einer «japanischen» D2-Lymphadenektomie (mit Splenektomie und Pankreasschwanzresektion) konnten bei vergleichbaren Tumorstadien keine Prognoseverbesserung durch eine D2-Lymphadenektomie beweisen [11, 12]. Die Frage, ob eine D2-Lymphadenektomie ohne Splenektomie und Pankreasschwanzresektion einer D1-Lymphadenektomie gleichwertig oder überlegen ist im Hinblick auf perioperative Morbidität und Mortalität sowie die Langzeitergebnisse, bleibt trotz dieser Studien weiter unbeantwortet. Selbstverständlich ist immer eine R0-Resektion anzustreben!

Ergebnisse der nordamerikanischen Intergroup Study (INT0116) [10] konnten zeigen, dass eine adjuvante Radiochemotherapie mit 5-Fluorouracil/Leucovorin und $4500 \mathrm{cGy}$ das mediane Überleben auf 36 Monate im Vergleich zu 27 Monaten in der Gruppe der nur beobachteten Patienten verlängert. Auch in der Langzeitbeobachtung konnte dieser Effekt bestätigt werden [13]. Allerdings war nur bei $10 \%$ der Patienten eine D2Lymphadenektomie und bei 36\% eine D1-Lymphadenektomie durchgeführt worden; bei der Mehrzahl der Patienten war eine D0-Dissektion durchgeführt worden, d.h., aus europäischer Sicht wurde eine inadäquate Chirurgie angewendet, die kein adäquates TNM-Staging erlaubt. Aus europäischer Sicht stellt die adjuvante Radiochemotherapie nach R0-Resektion eines Adenokarzinoms des Magens keinen neuen Standard dar.

Heike: Bei der Resektion von Magenkarzinomen sollte in jedem Fall mindestens eine D1-Lymphknotendissektion durchgeführt werden. Infolge der fehlenden adäquaten Lymphknotendissektion in der US-amerikanischen Intergroup-Studie zur adjuvanten Radiochemotherapie des Magenkarzinoms [10] bei über $50 \%$ der Patienten sind diese Studienergebnisse nicht auf Deutschland übertragbar. Die adjuvante Radiochemotherapie nach R0-Resektion von Magenkarzinomen mit D1/D2-Lymphknotendissektion stellt daher noch kein Standardverfahren dar.

Hohenberger: Für ein Magenkarzinom, das kein Frühkarzinom ist (d.h. Stadium II und III, T2 N0), ist die Gastrektomie mit D2-Lymphadenektomie erforderlich. Bei Sitz des Tumors im Antrum ist eine subtotale Gastrektomie gleichwertig zur Gastrektomie, zumindest wenn es sich um einen intestinalen Typ handelt. Beim diffusen Typ sollte auch beim jüngeren $\mathrm{Pa}-$ tienten gastrektomiert werden. Die D2-Lymphadenektomie sollte nur dann die Splenektomie beinhalten, wenn vom Tumorsitz her eine Lymphknotenmetastasierung in diese Region (suprapankreatische Lymphknoten- und Milzhiluslymphknoten) wahrscheinlich oder nachgewiesen ist. Hierzu kann das Maruyama-Computerprogramm herangezogen werden. Eine weitere positive Evaluation vorausgesetzt, kann eine «Sentinel-node»-Detektion ebenfalls dieses Ziel erfüllen. Die postoperative Radiochemotherapie ist bei einem derart beschriebenen chirurgischen Vorgehen kein Standard! Die US-amerikanische Intergroup-Studie [10] kann als Beleg in keiner Weise herangezogen werden. Eine randomisierte Studie Gastrektomie + D2-Lymphadenektomie +/- postoperative Radiochemotherapie ist dringend erforderlich.

Neuhaus: Für westliche Länder erscheint ein Vorteil einer höheren operativen Radikalität beim Magenkarzinom (D2- vs. D1-Lymphknotendissektion) nicht gesichert und kann somit nicht als Standard gelten. Wahrscheinlich profitieren allerdings Subgruppen von einer radikaleren Resektion. Eine adjuvante 
Radiochemotherapie ergibt für das Magenkarzinom in MetaAnalysen nur einen sehr geringen Vorteil hinsichtlich der Rezidiv-bedingten Mortalität gegenüber nicht adjuvant behandelten Patienten. Sie gilt somit nicht als Standard und bedarf einer Überprüfung unter einem europäischen Operationsstandard.

\section{Frage 5: Intensivierte adjuvante Chemotherapie beim Kolonkarzinom - gibt es einen neuen Goldstandard?}

Bechstein: Als Ergebnis der MOSAIC-Studie [14] konnte gezeigt werden, dass eine adjuvante Chemotherapie mit Folinsäure, 5-Fluorouracil und Oxaliplatin (FOLFOX) nach R0Resektion eines Kolonkarzinoms die Rate rezidivfreier Patienten nach 3 Jahren auf 79\% signifikant erhöht im Vergleich zu 73\% im Standardtherapiearm mit 5-Fluorouracil/Leucovorin. FOLFOX stellt damit einen neuen Standard in der adjuvanten Chemotherapie des Kolonkarzinoms dar.

Heike: Die MOSAIC-Studie [14] zeigte eine signifikant erhöhte Rate an krankheitsfreiem Überleben $(77,8 \%$ nach 3 Jahren) nach einer adjuvanten Chemotherapie mit FOLFOX gegenüber einer adjuvanten Therapie mit Folinsäure und 5Fluorouracil in gleicher Dosierung und Applikationsweise (72,9\%). Unter der Oxaliplatin-haltigen Chemotherapie wurden höhergradige Neuropathien bei etwa 12\% der Patienten beobachtet, die sich in den meisten Fällen nach 1 Jahr zurückbildeten. In Anbetracht dieser neuen Ergebnisse entspricht eine adjuvante Therapie von Patienten mit Stadium-III-Kolonkarzinomen mit dem FOLFOX-Protokoll neben den bisher eingesetzten Folinsäure/5-Fluorouracil-Therapieprotokollen einem neuen Standard in der adjuvanten Therapie bei Patienten mit Stadium-III-Kolonkarzinomen.

Hohenberger: Die neuen Substanzen (Oxaliplatin, Irinotecan) stellen eine wesentliche Bereicherung in der metastasierten Situation dar. Die Ansprechraten sind günstiger, die Überlebensraten auch. Dies wird allerdings durch eine höhere Toxizität erkauft, die hinsichtlich der Neurotoxizität des Oxaliplatin nicht reversibel ist. Für die adjuvante Situation liegen bisher nur vorläufige Berichte einer Studie [14] vor, so dass meines Erachtens eine Therapie hier nur innerhalb von Studien oder bei Hochrisiko-Patienten (z.B. Tumorzellen in der Peritoneal-Lavage) indiziert ist.

Neuhaus: Die kürzlich vorgestellten Ergebnisse der MOSAIC-Studie [14] ergaben eine statistisch signifikante Reduktion des Rezidivriskos eines komplett resezierten Kolonkarzinoms im Stadium II oder III durch die Addition von Oxaliplatin zur adjuvanten Standard-Chemotherapie. Diese Untersuchung und andere zum Teil noch laufende kontrollierte Studien sollten jedoch von den Fachgesellschaften bewertet werden, bevor ein neues Regime als «Goldstandard» allgemein empfohlen wird.

\section{Frage 6: Totale mesorektale Exzision (TME) - ein «Muss» beim Rektumkarzinom?}

Sauer: Neuere Studiendaten sprechen dafür, dass man durch kunstgerechte Durchführung der TME bei Karzinomen in allen Rektumdritteln die Rate lokoregionaler Rezidive deutlich senken kann. Dabei erlaubt die Dünnschicht-MRT der Tumorregion eine individuelle Therapieentscheidung. Allerdings muss die Qualität der TME durch den klinischen Pathologen durch akribische Untersuchungen beschrieben werden. Welche Konsequenzen aus der Tatsache, dass eine inkomplette TME durchgeführt wurde, zu ziehen sind, wurde in Studien bisher noch nicht untersucht.

Bechstein: Die systematische Durchführung der TME hat zu einem grundlegenden Wandel in den Ergebnissen der Chirurgie des Rektumkarzinoms geführt. Während ohne TME lokoregionäre Rezivraten von $15-45 \%$ beobachtet wurden, gelingt es mit TME, die Lokalrezidivrate unter $10 \%$ zu halten. Die TME ist ein «Muss» beim Rektumkarzinom.

Heike: TME ist ein «Muss» bei Rektumkarzinomen des mittleren und unteren Rektumdrittels, da dadurch die früher um $25 \%$ liegenden Lokalrezidivraten auf etwa 5-10\% abgesenkt werden können.

Hohenberger: Ja, solange der distale Tumorrand innerhalb von $12 \mathrm{~cm}$ oberhalb der Linea dentata liegt. Es sollten keine weiteren Einschränkungen wie etwa eine partielle TME akzeptiert werden, sonst wird hier der Kompromittierung der chirurgischen Technik Tür und Tor geöffnet.

Neuhaus: Soweit hierbei eine Beurteilung aus gastroenterologischer Sicht möglich ist, erscheinen die Vorteile und publizierten Studien so überzeugend, dass eine TME als Standardverfahren gelten sollte. Voraussetzung ist sicherlich ein präzises präoperatives Tumor-Staging, eine adäquate chirurgische Expertise sowie die Einbindung in multimodale Behandlungskonzepte.

\section{Frage 7: Was bringt die neoadjuvante Radiochemothera- pie, was die neoadjuvante Kurzzeitstrahlentherapie für den Patienten? Sind postoperative Komplikationen häufiger? Sind diese Konzepte der adjuvanten Therapie überlegen?}

Sauer: Zur Zeit stehen für Karzinome in den oberen zwei Rektumdritteln zwei Fraktionierungsschemata zur Verfügung: Die Kurzzeitvorbestrahlung mit 25 Gy in Einzeldosen von 5 Gy und die konventionell fraktionierte Bestrahlung mit 50 Gy in 6 Wochen. Dabei ist die Rolle der Chemotherapie im Rahmen der präoperativen Radiochemotherapie noch nicht ausreichend gesichert. Eine Sondersituation stellen Patienten mit 
tief sitzenden Tumoren und intendiertem Sphinktererhalt dar. Hier werden durch die neoadjuvante Radiochemotherapie die Bedingungen für eine kontinenzerhaltende Operation verbessert, und zwar wegen eines effektiven «down-sizing» und «down-staging», die mit einer Kurzzeitvorbestrahlung mit unmittelbar gefolgter Operation nicht zu erreichen sind. Die präoperative Radiochemotherapie ist der postoperativen (adjuvanten) hinsichtlich Verträglichkeit, der Ermöglichung kontinenzerhaltender Eingriffe und der Senkung der Lokalrezidivrate deutlich überlegen. Bei der präoperativen Kurzzeitvorbestrahlung sind signifikante Spätfolgen derzeit nicht auszuschließen.

Bechstein: Die neoadjuvante Radiochemotherapie beim Rektumkarzinom ermöglicht ein «down-staging» des Tumors und eine höhere Rate an Sphinkter-erhaltenden Operationen (Sauer, CAO/ARO/AIO-94 Studie: ausgewertet, Publikation in Vorbereitung). Eine präoperative Kurzzeitstrahlentherapie $(5 \times 5$ Gy) vor TME des Primärtumor senkt die Rate lokoregionärer Rezidive signifikant auf 2,4\% nach 2 Jahren gegenüber 8,2\% nach alleiniger Operation (TME) [15]. Postoperative Komplikationen nach neoadjuvanter Radiochemotherapie sind nicht signifikant erhöht [16]. Diese Konzepte sind der adjuvanten Therapie überlegen.

Heike: Die neoadjuvante Radiochemotherapie des Rektumkarzinoms scheint nach den Daten der deutschen CAO/ARO/ AIO-94-Studie (Sauer, Studie ausgewertet, Publikation in Vorbereitung) gegenüber der adjuvanten postoperativen Radiochemotherapie mit einer geringeren Rate an Anastomosenkomplikationen, einer höheren Chance auf Sphinkter-erhaltende Operationen (39 vs. 19\%) und einer geringeren Lokalrezidivrate (7 vs. $11 \%$ ) assoziiert zu sein.

Die präoperative hyperfraktionierte Kurzzeitbestrahlung ( $5 \times 5$ Gy) des Rektumkarzinoms verringert auch bei durchgeführter TME signifikant die Lokalrezidivrate (2,4 vs. 8,2\%; [15]), scheint jedoch keinen Einfluss auf die Überlebenswahrscheinlichkeit zu haben. Diese Therapieform führt im Gegensatz zur neoadjuvanten Radiochemotherapie nicht zu einer Tumorrückbildung vor Operation und damit nicht zu einer erhöhten Chance einer Sphinkter-erhaltenden Operation. Die Rate an perinealen postoperativen Komplikationen ist bei dieser Therapie leicht erhöht. Weiterhin scheinen funktionelle Störungen im Bereich des Enddarms und des Sphinkters längerfristig häufiger aufzutreten als bei Patienten nach primärer Operation (30 vs. 10\%; [17])

Hohenberger: Bei Patienten mit einem Rektumkarzinom T3/4 NX sollte eine präoperative Radiochemotherapie Standard sein. Ich würde dies auch für Patienten mit TX N+ empfehlen. Je fortgeschrittener der Tumor, desto eher ist eine konventionelle Langzeitstrahlentherapie indiziert. Der Stellenwert der Kurzzeittherapie kann noch nicht endgültig abgeschätzt werden. Entsprechende Patienten sollten in die in Deutschland gerade begonnene randomisierte Studie eingebracht werden. Die postoperative Komplikationsrate (Anastomoseninsuffizienz, Transfusionshäufigkeit) dürfte nach präoperativer Radiochemotherapie eindeutig höher liegen. Trotzdem ist dieses Konzept der adjuvanten, postoperativen Strahlentherapie im Hinblick auf die Dünndarmtoxizität überlegen. Häufig kann postoperativ nicht die volle Strahlendosis angebracht werden. Bei Patienten, die abdominell nicht voroperiert sind und bei denen durch entsprechende Lagerung die Dünndarmschlingen aus dem Strahlenfeld gehalten werden können, ist eine präoperative Strahlentherapie vorzuziehen.

Neuhaus: Die Vor- und Nachteile einer neoadjuvanten vs. einer adjuvanten Radiochemotherapie werden für das distale mobile Rektumkarzinom weiterhin kontrovers diskutiert. Bei fraglich R0-resektablen Tumoren sollte eine präoperative multimodale Therapie erfolgen. Der theoretische Vorteil einer Kurzzeitvorbestrahlung sollte in kontrollierten Studien geprüft werden. Die neoadjuvante Radiochemotherapie scheint nach den Ergebnissen großer Serien die postoperative Morbidität insgesamt nicht zu erhöhen und die Hospitalisationszeit nicht zu verlängern.

\section{Teilnehmer}

Prof. Dr. med. Wolf O. Bechstein

Klinik für Allgemein- und Gefäßchirurgie

Johann Wolfgang Goethe-Universität

Theodor-Stern-Kai 7, D-60590 Frankfurt

E-mailwolf.bechstein@kgu.de

PD Dr. med. Michael Heike

Medizinische Klinik Mitte

Gastroenterologie, Endokrinologie, Hämatologie und internistische Onkologie

Klinikum Dortmund gGmbH

Beurhausstraße 40, D-44137 Dortmund

E-mail stkd.med.gastroenterologie@dokom.net

Prof. Dr. med. Peter Hohenberger

Klinik für Chirurgie und Chirurgische Onkologie

Charité - Universitätsmedizin Berlin, Campus Buch

Robert-Rössle-Klinik im HELIOS Klinikum Berlin

Lindenberger Weg 80, D-13125 Berlin

E-mail hohenberger@rrk.charite-buch.de

Prof. Dr. med. Horst Neuhaus

Medizinische Klinik

Evangelisches Krankenhaus Düsseldorf

Kirchfeldstraße 40, D-40217 Düsseldorf

E-mail medizinischeklinik@evk-duesseldorf.de

Prof. Dr. med. Rolf Sauer

Strahlenklinik

Universitätsklinikum Erlangen

Postfach 23 06, D-91012 Erlangen

E-mail sekretariat@strahlen.imed.uni-erlangen.de 


\section{Literatur}

1 Ortner MA, Dorta G, Blum AL, Michetti P: Endoscopic interventions for preneoplastic and neoplastic lesions: Mucosectomy, argon plasma coagulation, and photodynamic therapy. Dig Dis 2002;20: 167-172.

2 Yagi K, Nakamura A, Sekine A: Accuracy of magnifying endoscopy with methylene blue in the diagnosis of specialized intestinal metaplasia and shortsegment Barrett's esophagus in Japanese patients without Helicobacter pylori infection. Gastrointest Endosc 2003;58:189-195.

3 Kouklakis GS, Kountouras J, Dokas SM, Molyvas EJ, Vourvoulakis GP, Minopoulos GI: Methylene blue chromoendoscopy for the detection of Barrett's esophagus in a Greek cohort. Endoscopy 2003;35:383-387.

4 Kelly MD, Walsh TN: Adjuvant treatment for resectable adenocarcinoma of the oesophagus. ANZ J Surg 2003;73:976-978.

5 El Nakadi I, Van Laethem JL, Houben JJ, Gay F, Closset J, Van Houtte P, Danhier S, Limbosch JM, Lambilliotte JP, Gelin M: Squamous cell carcinoma of the esophagus: Multimodal therapy in locally advanced disease. World J Surg 2002;26:72-78.

6 Heise JW, Heep H, Frieling T, Sarbia M, Hartmann KA, Roher HD: Expense and benefit of neoadjuvant treatment in squamous cell carcinoma of the esophagus. BMC Cancer 2001;1:20.

7 Walsh TN, Noonan N, Hollywood D, Kelly A, Keeling $\mathrm{H}$, Hennessy TP: A comparison of multimodal therapy and surgery for esophageal adenocarcinoma. N Engl J Med 1996;335:462-467.
8 Bosset JF, Gignoux M, Triboulet JP, Tiret E, Mantion G, Elias D, Lozach P, Ollier JC, Pavy JJ, Mercier M, Sahmoud T: Chemoradiotherapy followed by surgery compared with surgery alone in squamouscell cancer of the esophagus. N Engl J Med 1997; 337:161-167.

9 Urba SG, Orringer MB, Turrisi A, Iannettoni M, Forastiere A, Strawderman M: Randomized trial of preoperative chemoradiation versus surgery alone in patients with locoregional esophageal carcinoma. J Clin Oncol 2001;19:305-313.

10 Macdonald JS, Smalley SR, Benedetti J, Hundahl SA, Estes NC, Stemmermann GN, Haller DG, Ajani JA, Dundersson LL, Jessup JM, Martenson JA: Chemoradiotherapy after surgery compared with surgery alone for adenocarcinoma of the stomach or gastroesophageal junction. N Engl J Med 2001:345:725-730

11 Bonenkamp JJ, Hermans J, Sasako M, van de Velde CJ: Extended lymph-node dissection for gastric cancer. Dutch Gastric Cancer Group. N Engl J Med 1999;340:908-914.

12 Cuschieri A, Weeden S, Fielding J, Bancewicz J, Craven J, Joypaul V, Sydes M, Fayers P: Patient survival after D1 and D2 resections for gastric cancer: Long-term results of the MRC randomized surgical trial. Surgical Co-Operative Group. Br J Cancer 1999;79:1522-1530.
13 Macdonald JS, Smalley SR, Benedetti J, Estes N, Haller DG, Ajani JA, Gunderson LL, Jessup M, Martenson JA: Postoperative combined radiation and chemotherapy improves disease-free survival and overall survival in resected adenocarcinoma of the stomach and gastroesophageal junction: Update of the results of Intergroup Study INT-0116 (SWOG 9008).ASCO 2004 Gastrointestinal Cancers Symposium, Upper GI Cancer (Esophageal and Gastric), abstract 6. www.asco.org.

14 De Gramont A, Banzi M, Navarro M, Tabernero J, Hishish T, Bridgewater J, Rivera A, Figer A, Fountzilas G, Andre T: Oxaliplatin/5-FU/LV in adjuvant colon cancer: Results of the international randomized MOSAIC trial. Proc SCO 2003;22:abstract 1015. www.asco.org.

15 Kapiteijn E, Marijnen CA, Nagtegaal ID, Putter H, Steup WH, Wiggers T, Ruttten HJ, Pahlman L, Glimelius B, van Krieken JH, Leer JW, van de Velde CJ, Dutch Colorectal Cancer Group: Preoperative radiotherapy combined with total mesorectal excision for resectable rectal cancer. N Engl J Med 2001;345:638-646.

16 Sauer R, Fietkau R, Wittekind C, Rodel C, Martus P, Hohenberger W, Tschmelitsch J, Sabitzer H, Karstens JH, Becker H, Hess C, Raab R, German Rectal Cancer Group: Adjuvant vs. neoadjuvant radiochemotherapy for locally advanced rectal cancer: the German trial CAO/ARO/AIO-94. Colorectal Dis 2003;5:406-415.

17 Dahlberg M, Glimelius B, Graf W, Pahlman L: Preoperative irradiation affects functional results after surgery for rectal cancer: Results from a randomized study. Dis Colon Rectum 1998;41:543-549. 